\title{
Finite-energy accelerating beam dynamics in wavelet-based representations
}

\author{
David Colas, ${ }^{1, *}$ Fabrice P. Laussy, ${ }^{2,3}$ and Matthew J. Davis $\odot^{1}$ \\ ${ }^{1}$ ARC Centre of Excellence in Future Low-Energy Electronics Technologies, School of Mathematics and Physics, \\ University of Queensland, St Lucia, Queensland 4072, Australia \\ ${ }^{2}$ Faculty of Science and Engineering, University of Wolverhampton, Wulfruna St, Wolverhampton WV1 1LY, United Kingdom \\ ${ }^{3}$ Russian Quantum Center, Novaya 100, 143025 Skolkovo, Moscow Region, Russia
}

(Received 18 December 2019; accepted 21 May 2020; published 15 June 2020)

\begin{abstract}
Accelerating beams are wave packets that appear to spontaneously accelerate without external potentials or applied forces. Since their first physical realization in the form of Airy beams, they have found applications on various platforms, spanning from optics to plasma physics. We investigate the dynamics of examples of finite-energy accelerating beams derived from catastrophe theory. We use a Madelung transformation in momentum space, combined with a wavelet transform analysis, to demonstrate that the beams' properties arise from a type of vanishing self-interference. We identify the modes responsible for the wave packet's acceleration, and we derive the general acceleration for higher-order cupsoid-related beams. We also demonstrate how bright solitons resulting from nonlinear Airy beams can be unambiguously detected using the wavelet transform. This methodology will allow for a better understanding of special wave packet dynamics.
\end{abstract}

DOI: 10.1103/PhysRevResearch.2.023337

\section{INTRODUCTION}

Accelerating beams are wave packet solutions of the free Schrödinger equation that possess the spectacular properties of accelerating without the need of an external potential and without diffracting. They were first discovered in 1979 by Berry and Balazs [1] with the Airy beam, a nonphysical solution that was long considered to be a mathematical curiosity. About three decades later, physical (square-integrable) approximations of Airy beams were experimentally demonstrated [2-4], in which the resulting Airy packets exhibited their special properties for a finite time. Finite-energy Airy beams (FEABs) have since been observed in platforms other than optically based ones, using, e.g., electron beams [5] or surface plasmon polaritons [6]. These realizations set the ground for a wide range of applications [7-12]. FEABs have also been extensively studied in a nonlinear context. In the presence of a self-focusing nonlinearity, the packet spontaneously splits between a weak accelerating remnant and an "off-shooting" bright soliton [13-19], bringing even richer physics and further potential applications.

More fundamentally, Airy beams are only the simplest example of a whole class of caustic beams that arise within the framework of catastrophe theory, introduced by Thom $[20,21]$. Caustic beams emerge from canonical diffraction

\footnotetext{
*d.colas@uq.edu.au

Published by the American Physical Society under the terms of the Creative Commons Attribution 4.0 International license. Further distribution of this work must maintain attribution to the author(s) and the published article's title, journal citation, and DOI.
}

integrals of codimension $K$,

$$
\xi_{K}(\boldsymbol{r})=\int_{-\infty}^{+\infty} e^{i V_{K}(u ; \boldsymbol{r})} d u
$$

with the associated potential functions,

$$
V_{K}(u ; \boldsymbol{r})=u^{K+2}+\sum_{n=1}^{K} r_{n} u^{n},
$$

which depend on one state variable $u$ and on certain control parameters $r_{n}$. For example, the fold catastrophe $(K=1)$ is related to the Airy function that is the FEAB's building block:

$$
\xi_{1}(x)=\int_{-\infty}^{+\infty} e^{i\left(u^{3}+u x\right)} d u=\frac{2 \pi}{\sqrt[3]{3}} \operatorname{Ai}\left(\frac{x}{\sqrt[3]{3}}\right),
$$

while higher-order catastrophes have also been recently studied. The cusp catastrophe $(K=2)$ was realized experimentally with Pearcey beams [22,23] and the swallowtail catastrophe $(K=3)$ with swallowtail beams [24].

In this paper we study finite-energy accelerating beam dynamics from a new perspective. We employ the wavelet transform (WT), a spectral decomposition which provides broad insights into nontrivial wave packets dynamics [25]. In the context of Schrödinger physics, this technique is particularly suited to detect interference between different wave packets [26]. It has been recently applied to understand the intriguing phenomenon of wave packet self-interference in exciton polaritons [27] and atomic condensates [28], and to explain the formation of nonlinear $\mathrm{X}$-waves in systems that possess a hyperbolic dispersion [29].

In the aforementioned cases, the wave dynamics arise due to the properties of the dispersion relation, such as its curvature or the presence of inflection points [27-29]. Accelerating beams are different. Their short time dynamics result from the intrinsic phase engineering of the initial condition combined 
with the effect of the parabolic dispersion relation. At long times the latter becomes dominant, and the packet reshapes into a smooth diffusing one. To understand the resulting complex wave packet phase dynamics, we use a Madelung decomposition of the wave function, not in real space, but in momentum space, which is here essential to interpret the results of the WT. We find that the accelerating fringes in the wave packet density result from a transient dynamical self-interference of the packet's internal modes. This allows us to derive the packet acceleration from the WT picture only, without the need for difficult calculations or special functions.

The paper is organized as follows. Section II introduces our method of analysis and we apply it to the FEAB. In Sec. III we study the case of a FEAB in the nonlinear regime. In Sec. IV we consider the case of the finite-energy Pearcey beam (FEPB), and we derive a general expression for the acceleration of higher-order cupsoid-related beams. Finally, Sec. V concludes the paper.

\section{FINITE-ENERGY AIRY BEAM DYNAMICS}

We start by introducing our method of analysis for a wave packet evolved with the one-dimensional Schrödinger equation, here written in momentum space:

$$
i \partial_{t} \psi(k, t)=E(k) \psi(k, t),
$$

with $\hbar=m=1$ and where the kinetic energy has the usual parabolic dispersion $E(k)=k^{2} / 2$. We consider a truncated Airy function as the initial condition, which can be expressed in momentum space as

$$
\psi_{0}(k)=\mathscr{F}_{k}\left[\operatorname{Ai}(b x) \exp \left(\left(a+i k_{0}\right) x\right)\right]=\frac{\exp \left(\frac{\left(a-i\left(k-k_{0}\right)\right)^{3}}{3 b^{3}}\right)}{2 b \sqrt{2 \pi}} .
$$

The parameter $b$ governs the width of the peaks in position space, while $a$ controls the exponential cutoff of the wave function density to ensure its square integrability [30]. The parameter $k_{0}$ specifies the momentum of the initial condition. The solution of Eq. (4) is obtained by simple integration: $\psi(k, t)=\psi_{0}(k) \exp \left(-i k^{2} t / 2\right)$. The real-space solution is found by inverse Fourier transform as $\psi(x, t)=$ $\mathscr{F}_{x}^{-1}[\psi(k, t)]$. The space-time dynamics for a FEAB with a negative initial momentum is shown in Fig. 1(a), and density profiles at selected times in Fig. 1(b). Because of the negative initial "kick", the center of mass of the packet moves to the left (dashed-blue line). However, and as expected, the Airy peaks accelerate along a parabolic trajectory, initially moving to the left, until a reversing time $t_{\text {rev }}$ after which they continue to accelerate to the right. With zero initial momentum $\left(k_{0}=0\right)$, the packet's center of mass would instead remain at the origin $(x=0)$ and the peaks would always move to the right.

Other representations of the wave function can also be accessed through the Fourier transform, such as $\psi(k, E)$ (often referred as the far field $)$ or $\psi(x, E)$ [31]. Alternatively, the WT permits a simultaneous representation of the wave function in both position $(x)$ and momentum $(k)$. The WT reads [25]

$$
\mathbb{W}(x, k)=(1 / \sqrt{|k|}) \int_{-\infty}^{+\infty} \psi\left(x^{\prime}\right) \mathcal{G}^{*}\left[\left(x^{\prime}-x\right) / k\right] d x^{\prime} .
$$
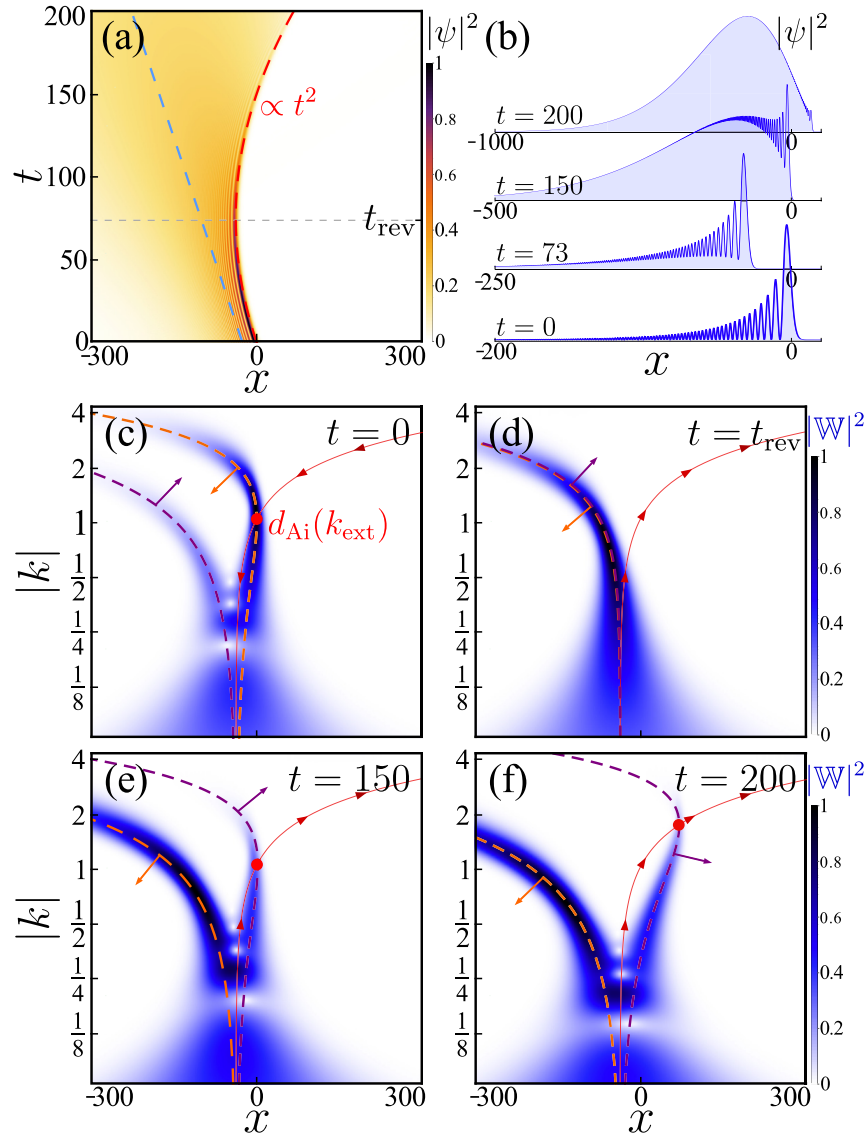

FIG. 1. Airy beam propagation. (a) Wave function density $|\psi(x, t)|^{2}$. The peaks initially move towards the left until $t=t_{\text {rev }}$. The dashed red, blue, and horizontal black lines indicate the wave front trajectory, the packet's center of mass $\bar{x}$, and the reversing time $t_{\text {rev }}$, respectively. (b) Wave function density $|\psi(x)|^{2}$ at selected times. (c)-(f) Corresponding wavelet energy densities $|\mathbb{W}(x, k)|^{2}$. The dashed purple and orange lines are the mode displacements $d_{\mathrm{Ai}}(k, t)$ derived from the FEAB solution's phase [see Eq. (9)]. The red dot indicates the position of the branch extremum $d_{\mathrm{Ai}}\left(k_{\mathrm{ext}}\right)$ around which the self-interference occurs and the solid red line shows its trajectory. Parameters are as follows: $a=0.01, b=0.3$, $k_{0}=-1, w_{\mathcal{G}}=8$. Supplemental Movie S1 provides an animation of the FEAB dynamics with its WT [40].

For this study we use the Gabor wavelet family,

$$
\mathcal{G}(x)=\sqrt[4]{\pi} \exp \left(i w_{\mathcal{G}} x\right) \exp \left(-x^{2} / 2\right),
$$

which consists of Gaussian functions with an internal frequency $w_{\mathcal{G}}$. We apply the WT to the FEAB and show its wavelet energy density $|\mathbb{W}(x, k)|^{2}$ at four selected times in Figs. 1(c)-1(f). It shows two distinct branches that are initially separated at $t=0$. However, the branches collapse onto each other at $t_{\text {rev }}$, before splitting and spreading at longer times. This peculiar distribution can be understood by analyzing the wave packet's phase dynamics.

It is common to perform a Madelung decomposition of the complex real-space wave function into an amplitude and a phase term as $\psi(x, t)=\sqrt{N(x, t)} \exp (-i \phi(x, t))$, notably to perform a hydrodynamic analysis [32]. In this picture, the gradient of the phase corresponds to the fluid velocity 
$v(x, t)=\partial_{x} \phi(x, t)$. Here, we perform the same decomposition of the FEAB, but in momentum space with $\psi(k, t)=$ $\sqrt{N(k)} \exp (-i \phi(k, t))$, where the amplitude is a timeindependent Gaussian of width $\sigma_{k}=\sqrt{3 b^{3} / 4 a}$, and where the phase is

$$
\phi(k, t)=\frac{3 a^{2}\left(k-k_{0}\right)-\left(k-k_{0}\right)^{3}}{3 b^{3}}+\frac{1}{2} k^{2} t .
$$

What does the gradient of the phase (with respect to $k$ ) represent? For a trivial Gaussian initial condition without any applied phase, the $k$-dependent phase of the packet would be simply $\phi(k, t)=E(k) t$. As the derivative of $E(k)$ gives the group velocity dispersion $v(k)$ [33], the gradient of the phase now represents a distance as $\partial_{k} \phi(k, t)=\partial_{k} E(k) t=v(k) t=$ $k t=d(k, t)$. Explicitly, the term $d(k, t)$ gives the distance traveled by a given mode $k$ after a time $t$. The simple case of the WT for a Gaussian packet is further discussed in Appendix A.

With a FEAB the modes propagate in a more complex fashion, with

$$
d_{\mathrm{Ai}}(k, t)=\partial_{k} \phi(k, t)=\frac{a^{2}-\left(k-k_{0}\right)^{2}}{b^{3}}+k t .
$$

From Eq. (9) we can see that $d_{\mathrm{Ai}}(k, t)$ contains two terms: The first comes from the FEAB's initial phase, and the second from the dispersion $E(k)$. The FEAB's dynamics arise from the interplay between these two phase terms, which govern the propagation of modes $k$. As only the second term in Eq. (9) is time dependent, at long times, the mode displacement essentially obeys the dispersion relation.

The mode displacement $d_{\mathrm{Ai}}(k, t)$ is plotted in Figs. 1(c)1(f) as dashed orange and purple lines on top of the wavelet energy density, and show excellent agreement with the branches from the WT calculations [34]. At long times, the mode displacement of the FEAB becomes essentially the one obtained from the dispersion (see Appendix A).

From the WT analysis, the presence of fringes in the realspace density can now be understood as self-interference of the wave packet. Indeed, $d_{\mathrm{Ai}}(k, t)$ is here a multivalued function [see Figs. 1(c)-1(f)], which leads to a self-interference when the wavelet energy density spreads over its extremum, i.e., where it becomes multivalued. For a given position $x$, two $k$ modes can have support in the wave function and overlap in real space, resulting in interference. This effect was first identified for condensed-matter systems possessing a nonparabolic dispersion relation, where the extrema correspond to inflection points of the dispersion [27,28].

The trajectory of the branch's extremum point, with coordinates $\left\{d_{\mathrm{Ai}}\left(k_{\text {ext }}\right), k_{\text {ext }}\right\}$ in the $x$ - $k$ phase space, can be determined from the expression of $d_{\mathrm{Ai}}(k, t)$. First, solving $\partial_{k} d_{\mathrm{Ai}}(k, t)=0$ for $k$, one obtains $k_{\text {ext }}=\frac{1}{2}\left(2 k_{0}+b^{3} t\right)$. One can then substitute $k_{\text {ext }}$ back into Eq. (9), which gives

$$
d_{\mathrm{Ai}}\left(k_{\mathrm{ext}}\right)=\frac{a^{2}}{b^{3}}+k_{0} t+\frac{b^{3} t^{2}}{4} .
$$

The point $\left\{d_{\mathrm{Ai}}\left(k_{\text {ext }}\right), k_{\text {ext }}\right\}$ is shown in Figs. 1(c)-1(f) as a red dot and its trajectory as a solid red line. As $d_{\mathrm{Ai}}\left(k_{\mathrm{ext}}\right)$ corresponds to the largest mode displacement, it gives, from this simple calculation, the trajectory of the FEAB wave front in real space. It is indeed parabolic and shown as a dashed red line in Fig. 1(a). One can also obtain the reversal time for the acceleration by solving $\partial_{t} d_{\mathrm{Ai}}\left(k_{\mathrm{ext}}\right)=0$ for $t$, which gives $t_{\mathrm{rev}}=-2 k_{0} / b^{3}$.

In the systems with a nonparabolic dispersion mentioned earlier [27,28], the value $k_{\text {ext }}$ is time independent, i.e., the self-interference always occurs around the same value of momentum. What makes the FEAB special is the fact that both the coordinates of the point $\left\{d_{\mathrm{Ai}}\left(k_{\mathrm{ext}}\right), k_{\mathrm{ext}}\right\}$ around which the self-interference occurs are time dependent. This explains why the FEAB's density fringes vanish at long times. One can observe from Figs. 1(c)-1(f) that the wavelet energy density distribution along $k$ is roughly constant in time-it mostly spreads along $x$. However, the point $\left\{d_{\mathrm{Ai}}\left(k_{\mathrm{ext}}\right), k_{\mathrm{ext}}\right\}$ linearly shifts to large momentum as $k_{\text {ext }} \propto t$. At long times, there is less and less signal available to participate into the self-interference effect, which explains why the wave packet's fringes inevitably disappear. One could maintain the selfinterference for a longer time by reducing the exponential cutoff $a$ to increase the spread in momentum space, since $\sigma_{k} \propto$ $1 / \sqrt{a}$. Alternatively, in Appendix B we discuss the possibility of maintaining the self-interference by engineering additional mode filtering and amplification.

\section{FINITE-ENERGY AIRY BEAM DYNAMICS WITH SELF-FOCUSING NONLINEARITY}

We now briefly consider the effects of introducing an attractive interaction on the FEAB dynamics, rewriting Eq. (4) as a $1 \mathrm{D}$ Gross-Pitaevskii equation:

$$
i \partial_{t} \psi(x, t)=\left[-\frac{1}{2} \partial_{x}^{2}+g|\psi(x, t)|^{2}\right] \psi(x, t),
$$

where $g<0$ is the interaction strength. We solve Eq. (11) for the same parameters as in Sec. II, and show the wave packet dynamics in Figs. 2(a) and 2(b).

In this situation, the wave front follows a trajectory that slightly deviates from a parabola [35], along with the generation of a density peak propagating at constant velocity, previously identified as an "off-shooting" bright soliton [13-19]. This can be confirmed by looking at the far field $|\psi(k, E)|^{2}$, plotted in Fig. 2(c). It follows the usual noninteracting parabolic dispersion, but also displays a clear linear dispersion, tangential to the parabola at the point $k=k_{0}$, which is an explicit signature of a bright soliton [36,37]. The bright soliton dispersion is obtained by performing a Taylor expansion of the main (parabolic) branch around $k_{0}$, giving

$$
E_{\mathrm{BS}}(k)=k k_{0}-k_{0}^{2} / 2 \text {. }
$$

The bright soliton velocity can be determined from the slope of the linear dispersion as $v_{\mathrm{BS}}=\partial_{k} E_{\mathrm{BS}}(k)=k_{0}$. In the wavelet analysis, this corresponds to a single mode displacement:

$$
d_{\mathrm{BS}}(k, t)=\partial_{k} E_{\mathrm{BS}}(k) t=k_{0} t,
$$

which appears as a vertical line in the $x-k$ representation, as shown in Fig. 2(d). As the bright soliton arises from a nondiffusing mode, i.e., a linear dispersion, its wavelet energy density remains localized around the point $\left\{k_{0} t, k_{0}\right\}$. This illustrates how the WT can be used to detect the bright 

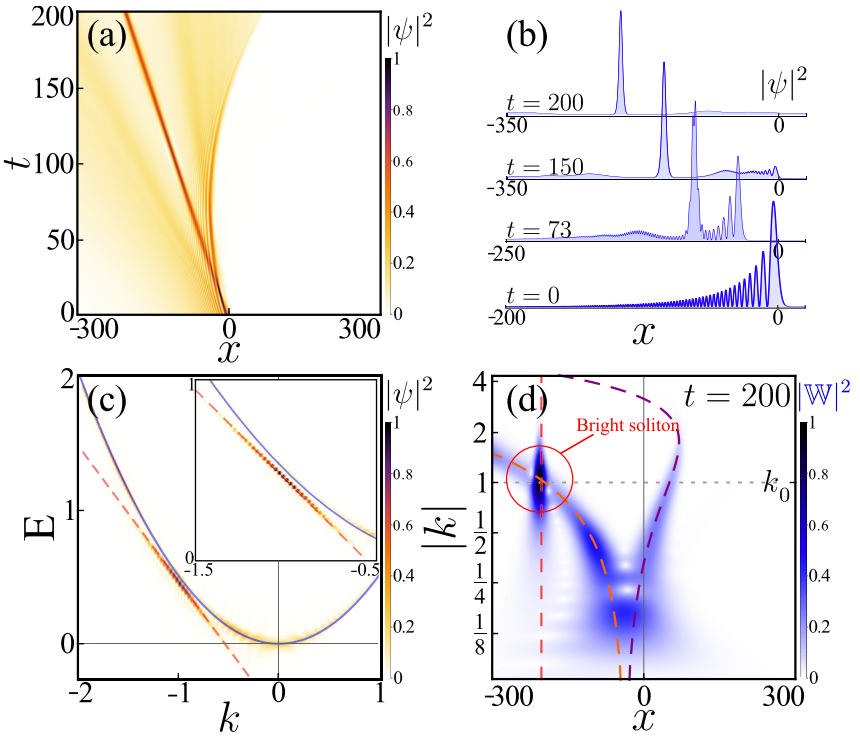

FIG. 2. Airy beam propagation with self-focusing nonlinearity $g=-0.5$ and initial momentum $k_{0}=-1$. (a) Wave function density $|\psi(x, t)|^{2}$. (b) Wave function density $|\psi(x)|^{2}$ at selected times. (c) Spectral density $|\psi(k, E)|^{2}$ lying on the parabolic dispersion $E(k)$ (blue line), and the bright soliton dispersion $E_{\mathrm{BS}}(k)$ (dashed red line). (Inset) Close up of the bright soliton dispersion. (d) Wavelet energy density $|\mathbb{W}(x, k)|^{2}$ at $t=200$. The vertical dashed red line stands for the mode displacement associated with the bright soliton dispersion $E_{\mathrm{BS}}(k)$. Supplemental Movie S2 provides an animation of the nonlinear FEAB dynamics with its WT [40].

soliton signature and to distinguish it from the remnant beam, complementary to the usual Fourier techniques.

\section{FINITE-ENERGY PEARCEY BEAM DYNAMICS}

We now consider the case of the FEPB obtained from the cusp catastrophe $(K=2)$ :

$$
\xi_{2}(x, y)=\int_{-\infty}^{+\infty} e^{i\left(u^{4}+u^{2} y+u x\right)} d u=\operatorname{Pe}(x, y),
$$

which defines the Pearcey function [38]. The initial condition for Eq. (4) is now, in one dimension, $\psi_{0}(x)=$ $\operatorname{Pe}(x, 0) \exp \left(-\sigma x^{2}\right)$. The square integrability is ensured by the Gaussian term of width $\sigma$ which cuts off the "fat" tails of $\operatorname{Pe}(x, 0)$. The Pearcey function can be expressed in momentum space as $\mathscr{F}_{k}[\mathrm{Pe}(x, 0)]=2 \pi \exp \left(i k^{4}\right)$ [39]. The full solution of Eq. (4) for a FEPB reads $\psi(k, t)=$ $2 \pi \exp \left[-i\left(-k^{4}+k^{2} t / 2\right)\right]$, and typical Pearcey beam dynamics is shown in Figs. 3(a) and 3(b). Following the procedure previously applied to the Airy beam, one obtains the mode propagation distance $d_{\mathrm{Pe}}(k, t)=-4 k^{3}+k t$ and hence the extremum mode $k_{\text {ext }}= \pm \sqrt{t / 12}$. Subsequently, the wave front trajectories are

$$
d_{\mathrm{Pe}}\left(k_{\mathrm{ext}}\right)= \pm(t / 3)^{3 / 2} .
$$

Here a double self-interference effect takes place as $d_{\mathrm{Pe}}(k, t)$ is multivalued for both branches. The interference disappears as the points $\left\{d_{\mathrm{Pe}}\left(k_{\mathrm{ext}}\right), k_{\mathrm{ext}}\right\}$ drift away from the wavelet energy density as shown in Figs. 3(c)-3(f).
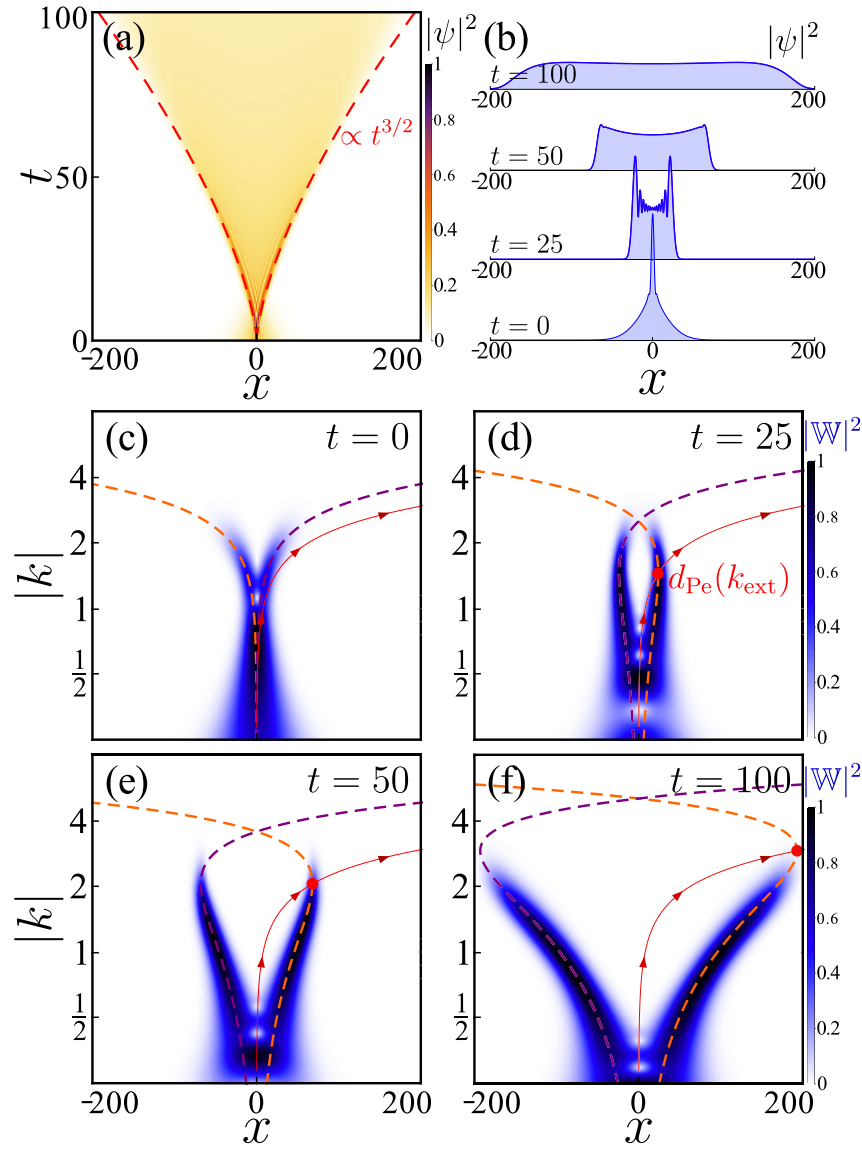

FIG. 3. Pearcey beam propagation. (a) Wave function density $|\psi(x, t)|^{2}$. (b) Wave function density $|\psi(x)|^{2}$ at selected times. (c)(f) Corresponding wavelet energy densities $|\mathbb{W}(x, k)|^{2}$. The dashed purple and orange lines are the mode displacements $d_{\mathrm{Pe}}(k, t)$ derived from Eq. (9). The red dot indicates the position of one branch extremum $d_{\mathrm{Pe}}\left(k_{\mathrm{ext}}\right)$ around which the self-interference occurs, and the solid red line shows its trajectory. Parameters are as follows: $\sigma=1 / 1000$. Supplemental Movie S3 provides an animation of the FEPB dynamics with its WT [40].

Unlike the FEAB [see Eq. (10)], the two FEPB wave fronts accelerate as $t^{3 / 2}$ and not as $t^{2}$, which is a consequence of the phase factor imprinted in the initial condition: $\sim \exp \left(i k^{4}\right)$ for the FEPB vs $\sim \exp \left(i k^{3}\right)$ for the FEAB. We can generalize our previous result for an initial phase containing any power of $k$. Considering a packet with a phase proportional to $\exp \left(i k^{n}\right)$ and following the same method as before, one can find the generalized wave front acceleration,

$$
d\left(k_{\mathrm{ext}} ; n\right)=n(n-2)\left(\frac{t}{n(n-1)}\right)^{\frac{n-1}{n-2}},
$$

which is a $t^{2}$ and $t^{3 / 2}$ acceleration for $n=3$ and 4 . In the limit of large $n$, one reaches a nonaccelerating limit as $d\left(k_{\text {ext }} ; n \rightarrow\right.$ $\infty)=t$.

\section{CONCLUSION}

In conclusion, we have shown that the dynamics of finiteenergy accelerating beams can be fully understood from a careful phase dynamics analysis using the WT and a 

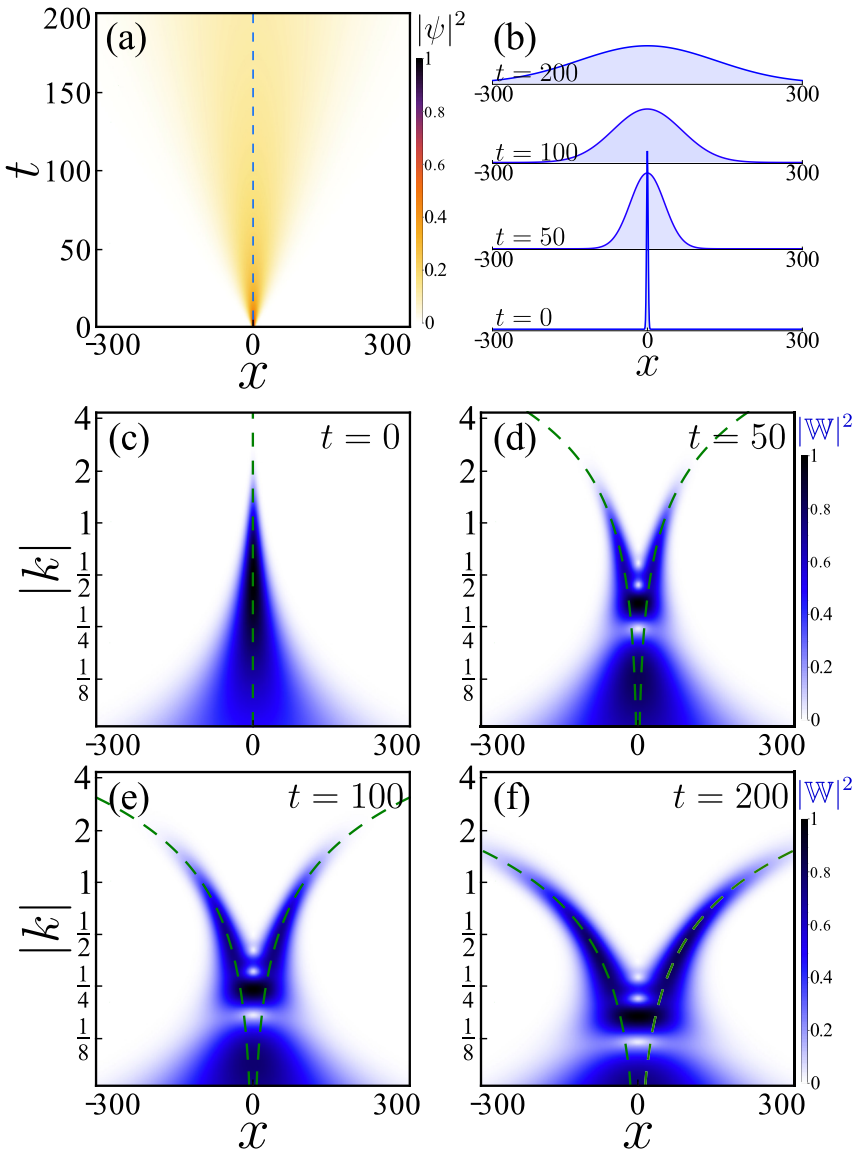

FIG. 4. Diffusion of an initially sharp $(\sigma=1)$ Gaussian wave packet. (a) Wave function density $|\psi(x, t)|^{2}$. The dashed blue line indicates the packet's center of mass $\bar{x}$. (b) Density profile at selected times. (c)-(f) Corresponding wavelet energy density $|\mathbb{W}(x, k)|^{2}$. The dashed green lines indicate the displacement $d(k)$ of mode $k$ derived from the parabolic dispersion relation. Supplemental Video $\mathrm{S} 4$ provides an animation of the Gaussian packet dynamics with its WT [40].

Madelung decomposition. We have identified that the key properties of the beams arise from a transient self-interference of the wave packet, and that the wave front acceleration can be obtained analytically. We find that the reshaping mechanism that controls the accelerating wave front originates from a dynamical shift of the extremum mode, towards of regions of low spectral density. This method of analysis is applicable to other accelerating beams with different phase engineering, as well as nonlinear objects like bright solitons with a welldefined dispersion.

\section{ACKNOWLEDGMENTS}

This research was supported by the Australian Research Council Centre of Excellence in Future Low-Energy Electronics Technologies (Project No. CE170100039) and by the Australian government. It was also supported by the Ministry of Science and Education of the Russian Federation through the Russian-Greek Project No. RFMEFI61617X0085 and the Spanish MINECO under Contract No. FIS2015-64951-R (CLAQUE).
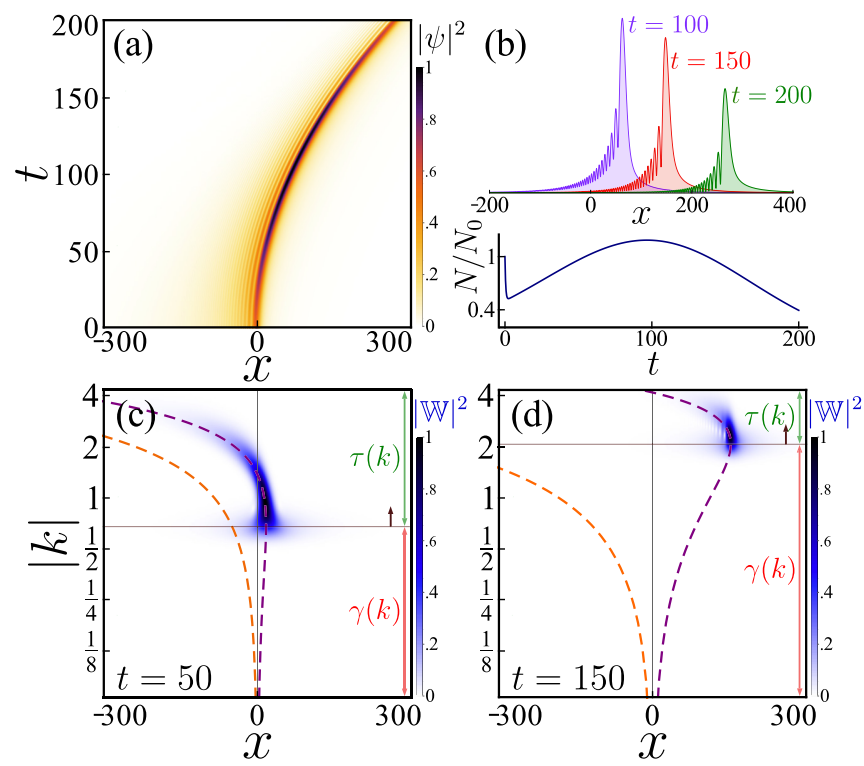

FIG. 5. Airy-engineered wave front. (a) Wave function density $|\psi(x, t)|^{2}$. (b) Wave function density $|\psi(x)|^{2}$ at selected times (top) and evolution of the total normalized population (bottom). (c) and (d) Wavelet energy density $|\mathbb{W}(x, k)|^{2}$ at two selected times. The left branch is fully damped from $t=0$ and the right branch is progressively damped, following the point $\left\{d_{\mathrm{Ai}}\left(k_{\mathrm{ext}}\right), k_{\mathrm{ext}}\right\}$, while the rest of the signal is amplified. Supplemental Video S5 provides an animation of the engineered-FEAB dynamics with its WT [40].

\section{APPENDIX A: GAUSSIAN WAVE PACKET DYNAMICS}

We consider the solution of Eq. (4) for a Gaussian wave packet as the initial condition, which can be either written in real or momentum space as $\psi_{0}(k)=\mathscr{F}_{k}\left[\exp \left(-x^{2} / 2 \sigma_{x}^{2}\right)\right] \simeq$ $\exp \left(-k^{2} / 2 \sigma_{k}^{2}\right)$, with $\sigma_{x}=1 / \sigma_{k}$. The full solution in momentum space for Eq. (4) is

$$
\psi(k, t)=\exp \left(-\frac{i k^{2} t}{2}\right) \exp \left(-\frac{k^{2}}{2 \sigma_{k}^{2}}\right) .
$$

In Fig. 4(a) we show an example of the well-known freely diffusing Gaussian wave packet, initialized with $\sigma_{x}=1$, as seen in the density profiles in Fig. 4(b). The dashed blue line here shows the position of the packet's center of mass. We apply the WT to the diffusing Gaussian packet and show the wavelet energy density $|\mathbb{W}(x, k)|^{2}$ at selected times in Figs. 4(c)-4(f). It is initially tightly distributed around $x=0$ where the packet stands, and then spreads as two branches. As in Sec. II, we decompose the complex momentum-space wave function into an amplitude term and a phase term as $\psi(k, t)=\sqrt{N(k)} \exp (-i \phi(k, t))$, with the amplitude being

$$
\sqrt{N(k)}=\exp \left(-\frac{k^{2}}{2 \sigma_{k}^{2}}\right),
$$

and the phase,

$$
\phi(k, t)=E(k) t=k^{2} t / 2 .
$$

We now compute the gradient of the phase (with respect to $k$ ):

$$
d(k, t)=\partial_{k} \phi(k, t)=\partial_{k} E(k) t=v(k) t=k t .
$$


As the $k$-dependent velocity is obtained by taking the derivative of the dispersion relation, the gradient of the phase in momentum space represents a distance $d(k, t)$ of propagation of a given mode $k$ at a time $t$. The distance traveled for each mode of the wave packet is superimposed on the wavelet energy density shown in Figs. 4(c)-4(f). We note that this result would be qualitatively comparable to other non-Gaussian wave packets evolved on the same parabolic dispersion, as long as they do not initially contain any complex phase relationships. This case corresponds to the long times limit of finite-energy Airy beams shown in Fig. 1.

\section{APPENDIX B: FINITE-ENERGY AIRY BEAM DYNAMICS WITH DYNAMICAL MODE FILTERING/AMPLIFICATION}

From the wavelet spectra in Fig. 1 it can be seen that, at long times, the energy density around $\left\{d_{\mathrm{Ai}}\left(k_{\text {ext }}\right), k_{\text {ext }}\right\}$ is small. The majority of the signal comes from modes participating in the reshaping of the packet. Therefore we consider developing a scheme to damp out those modes, and enhance those contributing to the self-interference, and hence to the accelerating peaks. We set up a dynamical high-pass filter to damp all the modes below a certain momentum close to $k_{\text {ext }}$. This can be translated as a momentum and time-dependent loss term for the Schrödinger equation. Similarly, we set a dynamical amplification for the remaining modes with a gain term, in order to limit the decay of the normalization. We can now rewrite Eq. (4) as an open-dissipative Schrödinger equation,

$$
i \partial_{t} \psi(k, t)=\left[\frac{k^{2}}{2}+i(\tau(k, t)-\gamma(k, t))\right] \psi(k, t),
$$

where $\tau$ and $\gamma$ are the gain and loss terms, respectively. We define these functions as

$$
\begin{aligned}
& \gamma(k, t)=\Theta\left[-k+\frac{1}{2} b^{3} t\right], \\
& \tau(k, t)=\beta \Theta\left[k-\frac{1}{2} b^{3} t\right],
\end{aligned}
$$

where $\Theta$ is the Heavyside step function, and $\beta$ a constant chosen to control the amplification of the remaining modes. The "boundary" in $k$ space between damping and amplification here follows the position of $d_{\mathrm{Ai}}\left(k_{\text {ext }}\right)$, which is a linear function of time.

To solve Eq. (B1) we use the same parameters as in Fig. 1 but with $k_{0}=0$. The newly Airy-engineered wave packet is shown in Figs. 5(a) and 5(b) and does not display any reshaping. Instead, it is an essentially shape-preserving and accelerating wave front. The normalization is approximately constant over the considered time interval due to the amplification of the high $k$ modes. The effect of the high-pass filter is shown in Figs. 5(c) and 5(d). The signal overlapping the left branch of $d_{\mathrm{Ai}}(k, t)$ is fully damped from $t=0$, leading to a sudden population decrease at short times. The signal overlapping the right branch is then progressively damped, following the drift of $d_{\mathrm{Ai}}\left(k_{\mathrm{ext}}\right)$, while the remaining signal is amplified.

This is the simplest way to dynamically damp and amplify a desired range of modes. This is sufficient to prevent the total population varying by more than a factor of two over the time interval we consider. This procedure could be further optimized using more complex functions for $\tau(k, t)$ and $\gamma(k, t)$, notably to enable an experimental realization of this scheme.
[1] M. V. Berry and N. L. Balazs, Am. J. Phys. 47, 264 (1979).

[2] G. A. Siviloglou, J. Broky, A. Dogariu, and D. N. Christodoulides, Phys. Rev. Lett. 99, 213901 (2007).

[3] G. A. Siviloglou and D. N. Christodoulides, Opt. Lett. 32, 979 (2007).

[4] T. Ellenbogen, N. Voloch-Bloch, A. Ganany-Padowicz, and A. Arie, Nat. Photon. 3, 395 (2009).

[5] N. Voloch-Bloch, Y. Lereah, Y. Lilach, A. Gover, and A. Arie, Nature (London) 494, 331 (2013).

[6] P. Zhang, S. Wang, Y. Liu, X. Yin, C. Lu, Z. Chen, and X. Zhang, Opt. Lett. 36, 3191 (2011).

[7] J. Baumgartl, M. Mazilu, and K. Dholakia, Nat. Photon. 2, 675 (2008).

[8] P. Polynkin, M. Kolesik, J. V. Moloney, G. A. Siviloglou, and D. N. Christodoulides, Science 324, 229 (2009).

[9] T. Vettenburg, H. I. C. Dalgarno, J. Nylk, C. Coll-Llado, D. E. K. Ferrier, T. Cizmar, F. J. Gunn-Moore, and K. Dholakia, Nat. Methods 11, 541 (2014).

[10] D. Abdollahpour, S. Suntsov, D. G. Papazoglou, and S. Tzortzakis, Phys. Rev. Lett. 105, 253901 (2010).

[11] Y. Gu and G. Gbur, Opt. Lett. 35, 3456 (2010).

[12] H. Nagar and Y. Roichman, Opt. Lett. 44, 1896 (2019).

[13] I. Kaminer, M. Segev, and D. N. Christodoulides, Phys. Rev. Lett. 106, 213903 (2011).
[14] A. Lotti, D. Faccio, A. Couairon, D. G. Papazoglou, P. Panagiotopoulos, D. Abdollahpour, and S. Tzortzakis, Phys. Rev. A 84, 021807(R) (2011).

[15] Y. Zhang, M. Belić, Z. Wu, H. Zheng, K. Lu, Y. Li, and Y. Zhang, Opt. Lett. 38, 4585 (2013).

[16] Y. Zhang, M. R. Belić, H. Zheng, H. Chen, C. Li, Y. Li, and Y. Zhang, Opt. Express 22, 7160 (2014).

[17] L. Zhang, P. Huang, C. Conti, Z. Wang, Y. Hu, D. Lei, and Y. L. D. Fan, Opt. Express 25, 1856 (2017).

[18] X. Zhang, D. Pierangeli, C. Conti, D. Fan, and L. Zhang, Opt. Express 26, 32971 (2018).

[19] T. Bouchet, N. Marsal, M. Sciamanna, and D. Wolfersberger, Phys. Rev. A 97, 051801(R) (2018).

[20] R. Thom, Stabilité Structurelle et Morphogénèse: Essai D’une Théorie Générale Des Modèles (W.A. Benjamin, Reading, 1972).

[21] M. V. Berry and C. Upstill, Prog. Opt. 18, 257 (1980).

[22] J. D. Ring, J. Lindberg, A. Mourka, M. Mazilu, K. Dholakia, and M. R. Dennis, Opt. Express 20, 18955 (2012).

[23] F. Zang, Y. Wang, and L. Li, Results Phys. 15, 102656 (2019).

[24] A. Zannotti, F. Diebel, M. Boguslawski, and C. Denz, New J. Phys. 19, 053004 (2017).

[25] L. Debnath and F. A. Shah, Wavelet Transforms and Their Applications, 2nd ed. (Birkhăuser, Basel, 2015). 
[26] C. H. Baker, D. A. Jordan, and P. M. Norris, Phys. Rev. B 86, 104306 (2012).

[27] D. Colas and F. P. Laussy, Phys. Rev. Lett. 116, 026401 (2016).

[28] D. Colas, F. P. Laussy, and M. J. Davis, Phys. Rev. Lett. 121, 055302 (2018).

[29] D. Colas, F. P. Laussy, and M. J. Davis, Phys. Rev. B 99, 214301 (2019).

[30] One cancompute the total normalization from Eq. (2) as $N=\int_{-\infty}^{+\infty}\left|\psi_{0}(x)\right|^{2} d x=\exp \left(\frac{2 a^{3}}{3 b^{3}}\right) / 8 \sqrt{2 \pi a b}$ and this result is finite.

[31] E. Wertz, L. Ferrier, D. D. Solnyshkov, R. Johne, D. Sanvitto, A. Lemaître, I. Sagnes, R. Grousson, A. V. Kavokin, P. Senellart, G. Malpuech, and J. Bloch, Nat. Phys. 6, 860 (2010).

[32] E. B. Sonin, Dynamics of Quantised Vortices in Superfluids (Cambridge University Press, Cambridge, 2016).

[33] C. Kittel, Introduction to Solid State Physics, 11th ed. (Wiley, New York, 1996).

[34] We note that the WT is governed by an uncertainty principle between its resolution in space $\left(\Delta_{x}\right)$ and momentum $\left(\Delta_{k}\right)$, so that the product $\Delta_{x} \Delta_{k}$ cannot be arbitrarily small. One typically needs to adapt the WT parameters such as the wavelet frequency to obtain a suitable resolution in the desired momentum/space range. In Figs. 1(c))-1(f) the WT is less adapted for the low momenta, which leads to broader energy distributions in this momentum range.

[35] Y. Hu, Z. Sun, D. Bongiovanni, D. Song, C. Lou, J. Xu, Z. Chen, and R. Morandotti, Opt. Lett. 37, 3201 (2012).

[36] O. A. Egorov, D. V. Skryabin, A. V. Yulin, and F. Lederer, Phys. Rev. Lett. 102, 153904 (2009).

[37] M. Sich, D. Krizhanovskii, M. Skolnick, A. Gorbach, R. Hartley, D. V. Skryabin, E. A. Cerda-Méndez, K. Biermann, R. Hey, and P. Santos, Nat. Photon. 6, 50 (2011).

[38] The function is named after Trevor Pearcey who, at the end of World War II, first obtained the numerical values for $\operatorname{Pe}(x, y)$ in a computational tour de force using a mechanical differential analyzer at the University of Cambridge.

[39] A closed form for $\mathscr{F}_{k}\left[\psi_{0}(x)\right]$, i.e., including the truncation, cannot be obtained in terms of simple functions. We thus based our analysis on the decomposition the infinite energy version of the beam $(\sigma=0)$ which we expect to be a good approximation for the FEPB, since the cutoff is chosen as $\sigma \ll 1$.

[40] See Supplemental Material at http://link.aps.org/supplemental/ 10.1103/PhysRevResearch.2.023337 for five videos, consisting of a time-animated version of Figs. 1-5. 